\title{
Characterization of Sleep Spindles Using Higher Order Statistics and Spectra
}

\author{
Tayfun Akgül*, Member, IEEE, Mingui Sun, Member, IEEE, Robert J. Sclabassi, Senior Member, IEEE, and \\ A. Enis Çetin, Member, IEEE
}

\begin{abstract}
This work characterizes the dynamics of sleep spindles, observed in electroencephalogram (EEG) recorded from humans during sleep, using both time and frequency domain methods which depend on higher order statistics and spectra. The time domain method combines the use of second- and third-order correlations to reveal information on the stationarity of periodic spindle rhythms to detect transitions between multiple activities. The frequency domain method, based on normalized spectrum and bispectrum, describes frequency interactions associated with nonlinearities occuring in the observed EEG.
\end{abstract}

Index Terms-Bispectrum, cumulants, EEG, higher order spectra, higher order statistics, sleep spindles, sum-of-cumulants.

\section{INTRODUCTION}

$\mathbf{T}$ HE electroencephalogram (EEG) is recorded from the scalp and reflects electrical activity of the brain. These signals are nonstationary and are possibly generated by underlying nonlinear processes [1]. This nonlinearity is not surprising since a wide range of physical and biologic systems show nonlinear behavior. The EEG is one of the bioelectric signals used in study of sleep state changes which are important in both basic and clinical neuroscience.

Adult human sleep, for which several models have been proposed [2], is classified into waking (W), quiet sleep (QS), and rapid eye movement (REM) stages. QS is further differentiated into four stages on the basis of brain, muscle, and eye activity. QS, REM, and occasional momentary wakings occur in a periodic sequence throughout the night, taking approximately $90 \mathrm{~min}$ in the adult. There is some suggestion that this alteration of $\mathrm{W}, \mathrm{QS}$, and REM is a manifestation of a basic rest/activity cycle characterized by periods of relative activity and action alternating with periods of relative inactivity and fantasy over the entire day.

The EEG is a continuous time-varying voltage, reflecting ongoing activity in the brain, normally recorded from the scalp in man [3]. EEG activity typically has amplitudes from 10 to

Manuscript received November 26, 1998; revised March 10, 2000. The work of T. Akgül was supported in part by TÜBİTAK-BAYG and Çukurova University, Turkey. The work of the M. Sun and R. J. Sclabassi was supported in part by the Whitaker Foundation and in part by the National Institutes of Health. Asterisk indicates corresponding author.

*T. Akgül is with TUBITAK Marmara Research Center, Information Technologies Research Institute, 41470 Gebze-Kocaeli, Turkey.

M. Sun and R. J. Sclabassi are with the Laboratory for Computational Neuroscience, Departments of Neurological Surgery and Electrical Engineering, University of Pittsburgh, Pittsburgh, PA 15261 USA.

A. E. Çetin is with the Department of Electrical Engineering, Bilkent University, Ankara, 06533 Turkey.

Publisher Item Identifier S 0018-9294(00)06413-2.
$100 \mu \mathrm{V}$ and a frequency content of from 0.5 to $40 \mathrm{~Hz}$. Signals of $10-30 \mu \mathrm{V}$ are considered low amplitude and potentials of $80-100 \mu \mathrm{V}$ are considered high amplitude. EEG, traditionally, is divided into four bands: $\delta$ from 0 to $4 \mathrm{~Hz}, \theta$ from 4 to $8 \mathrm{~Hz}, \alpha$ ranging 8 to $13 \mathrm{~Hz}$ and $\beta$ from 13 to $30 \mathrm{~Hz}$. An alert person displays a low amplitude EEG of mixed frequencies in the 13-18 $\mathrm{Hz}$ range, while a relaxed person produces large amounts of sinusoidal waves, at a single frequency in the $8-13 \mathrm{~Hz}$ range, which are particularly prominant at the back of the head. As an individual goes to sleep, alpha activity is replaced by a lower amplitude, mixed frequency voltage (stage 1 QS), which within minutes has superimposed 1- to 2-s bursts of 12 - to $14-\mathrm{Hz}$ activity called sleep spindles (stage 2 , QS). Several minutes later high-amplitude slow waves $(0.5-3 \mathrm{~Hz})$ appear and mark the onset of stage $3 \mathrm{QS}$. After about 10 min these slow waves dominate the EEG and the deepest stage of sleep, stage 4 is reached. After a return through these stages, REM sleep occurs, approximately $90 \mathrm{~min}$ after sleep onset.

Spindle activity can be considered as oscillations and noise-free sleep spindle waveforms may exhibit periodic, quasiperiodic or complex oscillations. Earlier studies [4], [5] have shown that there coexist two types of spontaneous spindle waves. In more recent work [6], it has been shown, by using matched filtering techniques, that one of these activities is centered around $12 \mathrm{~Hz}$ and the other around $14 \mathrm{~Hz}$. Some human and animal studies [7], [8] suggest that spindle and delta rhythms reciprocally oscillate creating difficulties in the detection of transitions between stages. In a recent study, Sun et al. [9] localized spindle activity in the brain via time-frequency analysis and synthesis of EEG, and showed that the origin of this activity is in the area of thalamus in humans, which is in agreement with previous data from the cat [5].

In this paper we apply higher order statistical measures both in the time and frequency domains to investigate the spindle activity associated with stage 2 sleep. The time domain techniques, which depend on the combination of second- and third-order statistics to trace the oscillatory dynamics of the waveforms around spindle activity, are used to investigate the nonstationary behavior of the spindles. While the frequency domain method is used to investigate frequency interactions which might be due to nonlinear properties of the central nervous system.

\section{METHODS}

Sleep spindles are considered to be periodic oscillations. These oscillations exhibit steady-state behavior which can be modeled either as a linear system with a sinusoidal input or as 
a nonlinear system with a limit cycle. Similarly, quasiperiodic oscillations [10] may also be linear oscillations where their system parameters may have more than one degree of freedom or they may be generated by a nonlinear system. On the other hand, complex oscillations which cannot be modeled by linear systems are due to nonlinearities having broader frequency distributions and sensitivity to initial conditions and system parameters [10]. Even seemingly very simple systems can exhibit complex behavior due to the nature of nonlinear components within these systems.

We review the methods, EEG acquisition and details of our algorithm in the following Sections.

\section{A. Second- and Third-order Statistics}

A well-established tool in the analysis of random or periodic signals is the estimate of autocorrelation function $R(m)$ (a second-order statistic)

$$
R(m)=\frac{1}{2 N+1} \sum_{n=-N}^{N} x(n) x(n+m)
$$

where $x(n)$ is a real, mean-removed, discrete-time signal of a windowed (stationary) EEG segment. The power spectrum of $x(n)$, which is the Fourier transform of the autocorrelation function

$$
P(\omega)=\sum_{m=-\infty}^{\infty} R(m) e^{-j \omega m}
$$

quantifies the power contents at the angular frequency $\omega$; however, it suppresses the phase relationships of these components. This loss of information can be important if there exists phase coupling due to nonlinearity in the signal of interest.

Phase information is conserved in non-Gaussian processes when the order of the spectrum is greater than two [11], [12]. The autotriplecorrelation function (third-order statistics) $c\left(m_{1}, m_{2}\right)$ of $x(n)$ is defined as [13], [14]

$$
c\left(m_{1}, m_{2}\right)=\frac{1}{2 N+1} \sum_{n=-N}^{N} x(n) x\left(n+m_{1}\right) x\left(n+m_{2}\right),
$$

and the 2-D Fourier transform of this equation, the bispectrum of the signal $x(n)$, is expressed by

$$
B\left(\omega_{1}, \omega_{2}\right)=\sum_{m_{1}=-\infty}^{\infty} \sum_{m_{2}=-\infty}^{\infty} c\left(m_{1}, m_{2}\right) e^{-j \omega_{1} m_{1}} e^{-j \omega_{2} m_{2}} .
$$

It can be shown [14] that the bispectrum in (4) can be written as

$$
B\left(\omega_{1}, \omega_{2}\right)=X\left(\omega_{1}\right) X\left(\omega_{2}\right) X^{*}\left(\omega_{1}+\omega_{2}\right)
$$

where * denotes complex conjugate, and $X(\omega)$ is the Fourier transform of $x(n)$. The power spectrum is given by

$$
P(\omega)=\frac{1}{M} X(\omega) X^{*}(\omega) .
$$

If the signal $x(n)$ is segmented into $M$ segments of equal length, or multichannel data is of interest, such as EEG, then the bispectrum and the power spectrum can be estimated by

$$
B\left(\omega_{1}, \omega_{2}\right)=\frac{1}{M} \sum_{i=1}^{M} X_{i}\left(\omega_{1}\right) X_{i}\left(\omega_{2}\right) X_{i}^{*}\left(\omega_{1}+\omega_{2}\right)
$$

and

$$
P(\omega)=\frac{1}{M} \sum_{i=1}^{M} X_{i}(\omega) X_{i}^{*}(\omega)
$$

where $X_{i}(\omega)$ is the Fourier transform of the $i$ th segment or the channel of the data. Equations (7) and (8) yield a normalized bispectrum (also referred to as bicoherence, second-order coherency, bicoherency index, etc.) [14]

$$
b^{2}\left(\omega_{1}, \omega_{2}\right)=\frac{\left|B\left(\omega_{1}, \omega_{2}\right)\right|^{2}}{P\left(\omega_{1}\right) P\left(\omega_{2}\right) P\left(\omega_{1}+\omega_{2}\right)}
$$

which can be an important and useful tool for the detection and characterization of nonlinearities [15], i.e., quadratically couplings.

If $x(n)$ is a periodic sequence with period $p$, then $R(m)$ is also periodic. Thus, $R(m)$ can be used to estimate the periodicity of the original signal. However, in speech analysis it is observed that the average magnitude difference function (AMDF) produces better results in estimating the fundamental period especially when the observed speech signal is "quasiperiodic." Since EEG signal is also quasiperiodic i.e., the periods may slightly vary in time, we use the AMDF function instead of the autocorrelation $R(m)$. AMDF is based on the following $k$ th-order difference [16]:

$$
d_{n}(k)=x(n)-x(n-k)
$$

which is zero for $k=0, \mp p, \mp 2 p \ldots$ when the signal $x(n)$ is truly periodic with period $p$. Now, by assuming that the sleep spindle segment of windowed EEG is periodic, we can use a function of $d(\cdot)$ to estimate the fundamental period

$\gamma_{n}(k)=\sum_{m=-\infty}^{\infty}|x(n+m) w(m)-x(n+m-k) w(m-k)|$

where $w(\cdot)$ is the window function and the range of $k$ is within the range of window support. This expression is referred to as the short-time average magnitude difference function [16]. One important feature of this function is that it is indeed similar to the short-time autocorrelation function (see [16, p. 149, and references therein]). AMDF is not only computationally simpler than the autocorrelation as only absolute differences are computed but it produces more prominent minima around $p, 2 p, \ldots$ compared to the autocorrelation. Note that, the lower and upper boundries of (11) should be arranged properly when the data is of finite length.

In the study of experimental data in this paper, we use the following modified version (mAMDF):

$$
D_{n}(k)=1-\frac{\gamma_{n}(k)}{\sigma_{\gamma}}
$$


which can be considered to be a second-order statistical measure, where $\sigma_{\gamma}=\sqrt{\Sigma_{i} \gamma_{n}^{2}(i)}$ is a normalization factor. ${ }^{1}$ Note that, similar to the autocorrelation function, the peak-to-peak values of $D_{n}(k)$ provide the fundamental period.

The inverse Fourier transform of the bispectrum $B\left(\omega_{1}, \omega_{2}\right)$, when calculated on one slice $\omega_{1}=\omega_{2}=\omega$, i.e.,

$$
\begin{aligned}
q(n) & =\frac{1}{2 \pi} \int_{-\pi}^{\pi} B(\omega, \omega) e^{j \omega n} d \omega \\
& =\sum_{m=-\infty}^{\infty} c(m, n-m), \quad-\infty<n<\infty
\end{aligned}
$$

is called the sum-of-autotriplecorrelation (SoA) [17]-[19] which is also periodic with period $p$ if $x(n)$ is periodic with period $p$. Clearly, the SoA in (13) is a third-order statistic which can be modified, in a similar fashion as in (11), as

$$
\varphi_{n}(k)=\sum_{m=-\infty}^{\infty}|q(n+m) w(m)-q(n+m-k) w(m-k)|
$$

and

$$
Q_{n}(k)=1-\frac{\varphi_{n}(k)}{\sigma_{\varphi}} .
$$

We call the modified AMDF of SoA, mAMDFSoA. Here, the term $\sigma_{\varphi}=\sqrt{\sum_{i} \varphi_{n}^{2}(i)}$ is, again, the normalization factor. Note that, since $q(n)$ in (13) can be truncated at certain $[-M, M]$ integer values, the upper and lower boundries of (15) should be arranged properly.

We use the measures based on the second- and third-order statistics [(12) and (15), respectively] together to estimate the periodicity of the spindle activity. If the spindle activity is purely periodic we expect these estimates to give similar results. ${ }^{2}$ If the results are not similar, then the existence of other linear or nonlinear relations are suggested. Moreover, the mAMDFSoA in (15) can reveal background frequency sources or the transition in signal even though other methods may under-estimate them.

\section{B. EEG Acquisition}

It is well known that the variation of the surface potential distribution on the scalp reflects functional activities emerging from the underlying brain [20]. This surface potential variation can be measured by affixing an array of electrodes, which are usually gold-plated, approximately $1 \mathrm{~cm}$ in diameter, to the scalp, and recording the voltages between pairs of these electrodes, which are then filtered, amplified, and stored. The resulting data is called the EEG. As previously described, sleep consists of states for which the brain waves are quite different. Spindles occur during stage two sleep, which occupies more than $50 \%$ of the total sleep time in a typical adult. The data studied here was spontaneous EEG (sampled at $256 \mathrm{~Hz}$ ) from three sleep-deprived male subjects aged 26, 28, and 39 years

${ }^{1}$ Alternatively, the standard deviation of the window can also be used for normalization.

${ }^{2}$ Similar or even identical results do not guarantee linearity. using a 64-channel amplification and acquisition system. To avoid aliasing, an analog bandpass filter with cutoff frequencies of $0.1 \mathrm{~Hz}$ and $70 \mathrm{~Hz}$ was utilized before digitization. Electrodes were placed at the sites defined in the International 10-20 System [21] and at the midpoints between these standard sites (Fig. 1).

\section{Estimation of $M A M D F$ and $m A M D F S o A$}

The following steps are applied to estimate $D_{n}(k)$ and $Q_{n}(k)$.

- mAMDF: A 256-point Hamming window is applied to the data. For $k=0,1, \ldots, 100, D_{n}(k)$ is estimated by using (11) and (12), respectively.

- mAMDFSoA: To estimate $Q_{n}(k)$ where $k=$ $0,1, \ldots, 100$, first the autotriplecorrelations in (3) and then sum-of-autocorrelations in (13) are employed. After applying a 256-point Hamming window to the estimated SoA sequence, (14) and (15) are used.

\section{Estimation of Normalized Bispectrum}

In general longer data is needed for meaningful bispectrum estimation [14]. We therefore prefer to group the EEG data over selected time segments (stages) for the frequency domain analysis. We take the simplest approach by constructing sets where the set members are frontal, central and parietal electrodes, i.e.,

$$
\begin{aligned}
& \text { set } \mathrm{C}=(\mathrm{Cz}, \mathrm{C} 1, \mathrm{C} 2, \mathrm{C} 3, \mathrm{C} 4, \mathrm{C} 5, \mathrm{C} 6), \\
& \text { set } \mathrm{F}=(\mathrm{Fz}, \mathrm{F} 1, \mathrm{~F} 2, \mathrm{~F} 3, \mathrm{~F} 4, \mathrm{~F} 5, \mathrm{~F} 6, \mathrm{~F} 7, \mathrm{~F} 8, \mathrm{~F} 9, \mathrm{~F} 10), \\
& \text { set } \mathrm{P}=(\mathrm{Pz}, \mathrm{P} 1, \mathrm{P} 2, \mathrm{P} 3, \mathrm{P} 4, \mathrm{P} 5, \mathrm{P} 6, \mathrm{P} 7, \mathrm{P} 8, \mathrm{P} 9) .
\end{aligned}
$$

While associating these neighboring electrodes, we visually evaluate the data. Moreover, we check for the results of commonly used second-order statistical classification methods ${ }^{3}$ such as correlation coefficients, ratio of harmonic energies, normalized bandwidths and mean frequencies [22]. Since approximately equivalent results are obtained, the channels are assumed to be associated adequately.

The estimation of the (averaged) normalized bispectrum is then accomplished for each group of channels sharing common features. These steps are itemized as follows.

- Apply a 256-point Hamming window to the EEG data to assure local stationarity.

- Remove the mean and estimate the bispectrum using (5).

- Repeat for all the members of the set and then average the bispectral values using (7).

- Estimate the power spectrum of each segment using (6) and average them in a similar fashion as in (8).

\footnotetext{
${ }^{3}$ Although selection based on the visual evaluation is relatively subjective, the statistical classifications are widely used in various applications. For example, the correlation coefficient is commonly used to measure the similarity between a pair of signals where electrode recordings which have close values can be good candidates for being in the same set. Similarly, the ratio of harmonic energy calculations is used to investigate the degree of difference between a signal and a sinusoid, hence the signals with high repetitiveness can be grouped together. The mean frequency and the normalized bandwidth correspond to the weighted average and the spread of the power spectrum, respectively.
} 


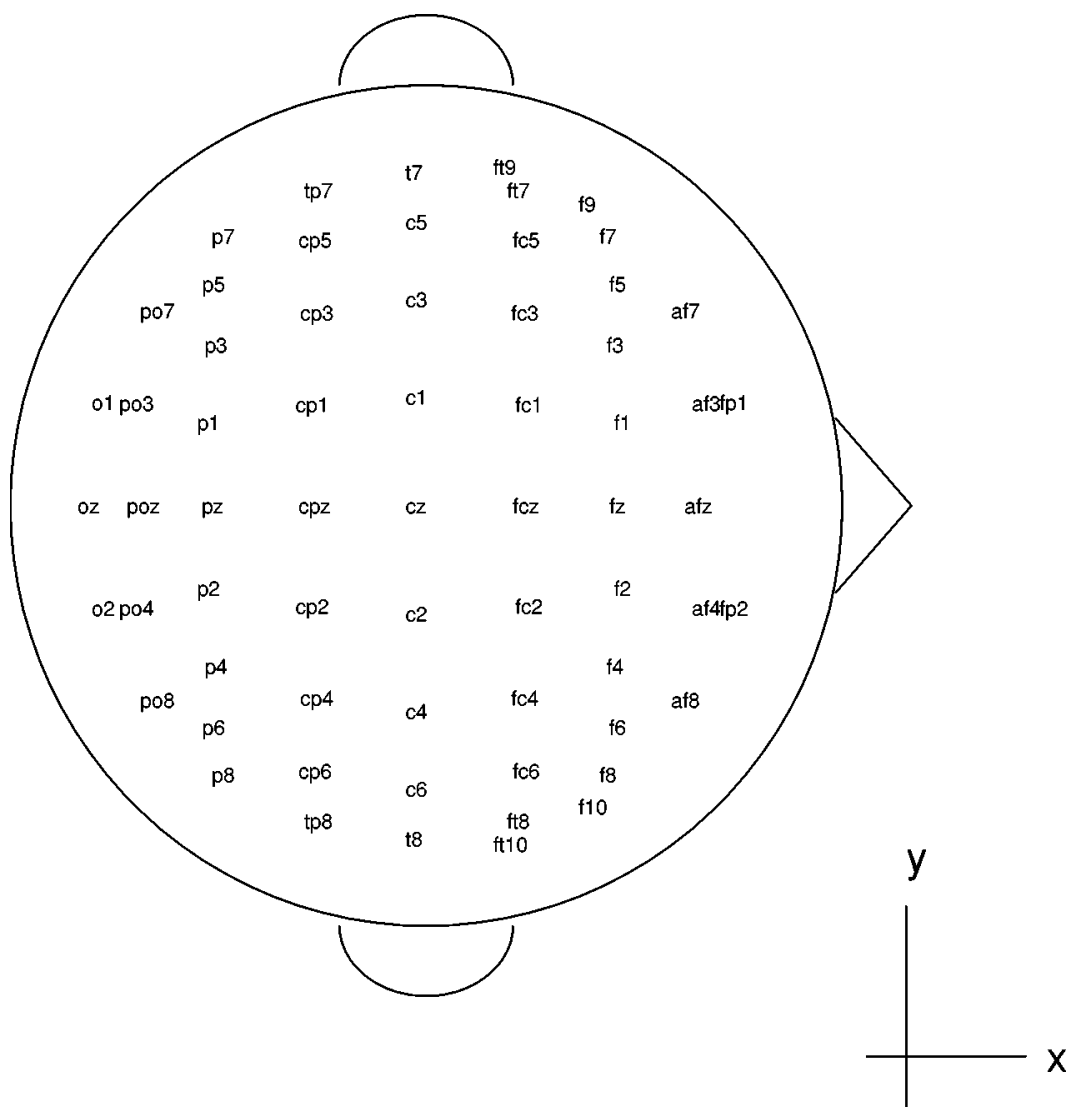

Fig. 1. Location of 64 recording electrodes utilized in obtaining the data in this paper.

- Mask the bispectral values below $10 \%$ of the maximum peak value. ${ }^{4}$

- Calculate the normalized bispectrum by using (9). Only the significant levels of normalized bispectrum $\left(b^{2}>\right.$ 0.1 ) are considered for the evaluation of energy interaction among frequencies.

It is important to note that, the results are obtained over one triangular region $\omega_{2}>0, \omega_{1}>\omega_{2}$ and $\omega_{1}+\omega_{2}<\pi$, simply because the bispectrum (normalized bispectrum) can be fully described over all frequencies by using the values in this region via its symmetry properties [13].

\section{RESULTS}

We present the time domain and frequency domain results separately.

\section{A. Time Domain Results}

We estimate $D_{n}(k)$ and $Q_{n}(k)$ for EEG recorded from $\mathrm{Cz}, \mathrm{Fz}$, $\mathrm{Pz}$, and Ozduring stage 2 quiet sleep. The top plots in Fig. 2(a)-(d)

\footnotetext{
${ }^{4}$ To identify a threshold for the bispectral estimate we apply a preliminary test which measures the "goodness" of the normalized bispectrum by using (100 sets of 512 points of) Gaussian data. The normalized bispectrum of Gaussian data is found to be relatively close to zero $\left(b^{2} \approx 0\right)$ where its maximum magnitude is measured to be less then of 0.1 . Therefore, $b^{2}>0.1$ is selected as the level of significance.
}

are segments of $8 \mathrm{~s}$ containing spindle activity plotted as a function of sample points. Both visual evalution and the energy distribution of bandpass (between 10-15 Hz) filtered data indicate that the spindle activity starts approximately at sample point 1000 and ends at 1400 as shown in the bottom plots in Fig. 2(a)-(d). Note that the energy distributions have greater values at the electrode position $\mathrm{Pz}$ conforming that the spindle activity can be observed clearly in the dorsal/posterior region of the head.

After applying a sliding window of 256 sample points (corresponds to $1 \mathrm{~s}$ in time) to the data, estimations were obtained for the mAMDF (12) and mADMFSoA (15). Interesting results are observed when the window is located between samples 1135 to $1390(\approx 4.5-5.5 \mathrm{~s}$ ); i.e., the time region where spindle activity is in progress as can be seen in the top plots of Fig. 3(a)-(d). We see that the second-order estimates (the middle plots of Fig. 3) are similar for all channels. This suggests that any second-order method (e.g., autocorrelation or power spectrum) will yield similar results in this segment for all channels. However, when we examine the third-order estimates (the bottom plots of Fig. 3), it may be seen that the results for $\mathrm{Oz}$ are radically different from all others, while the results for $\mathrm{Cz}$ and $\mathrm{Fz}$ are similar to each other. The third-order results for $\mathrm{Pz}$ are different from all other third-order results, but are similar to the second-order results for all other channels.

When the window is moved forward to cover samples 1198-1453 the second- and third-order estimates have similar results as shown in Fig. 4(a)-(d) consistent with the observed steady-state oscillation in all channels. 

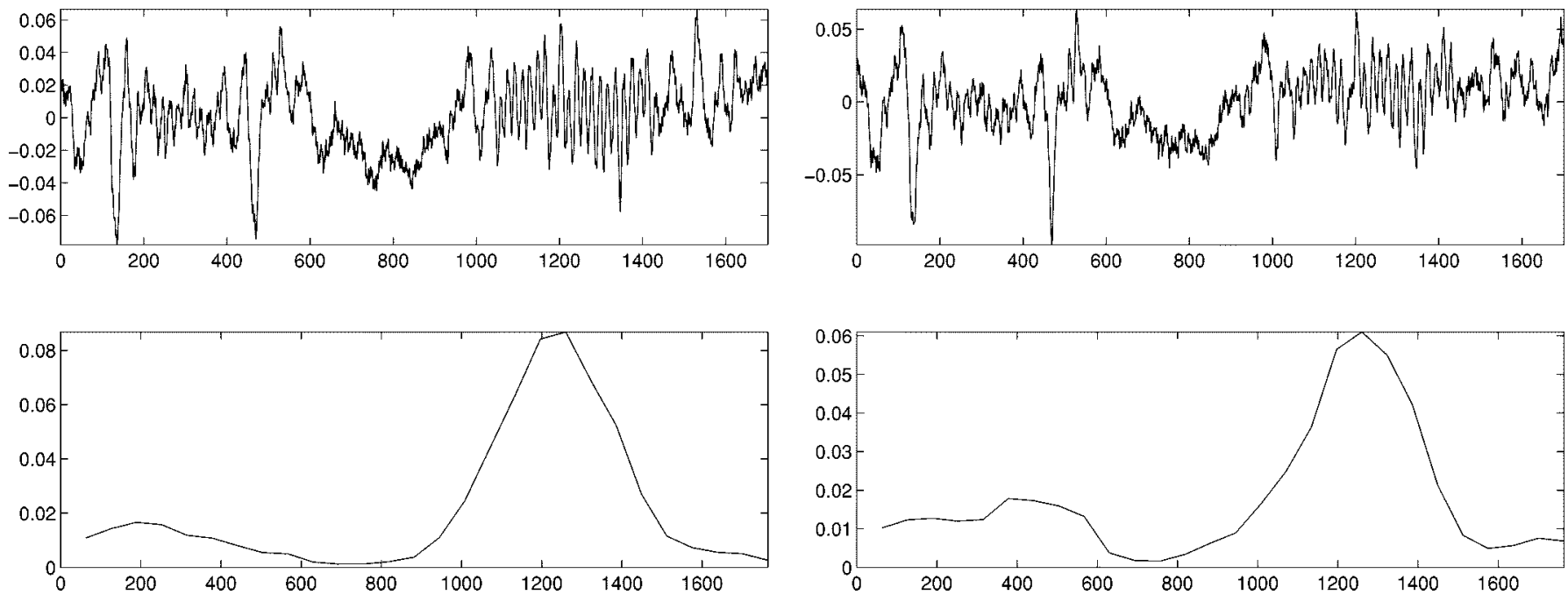

(a)
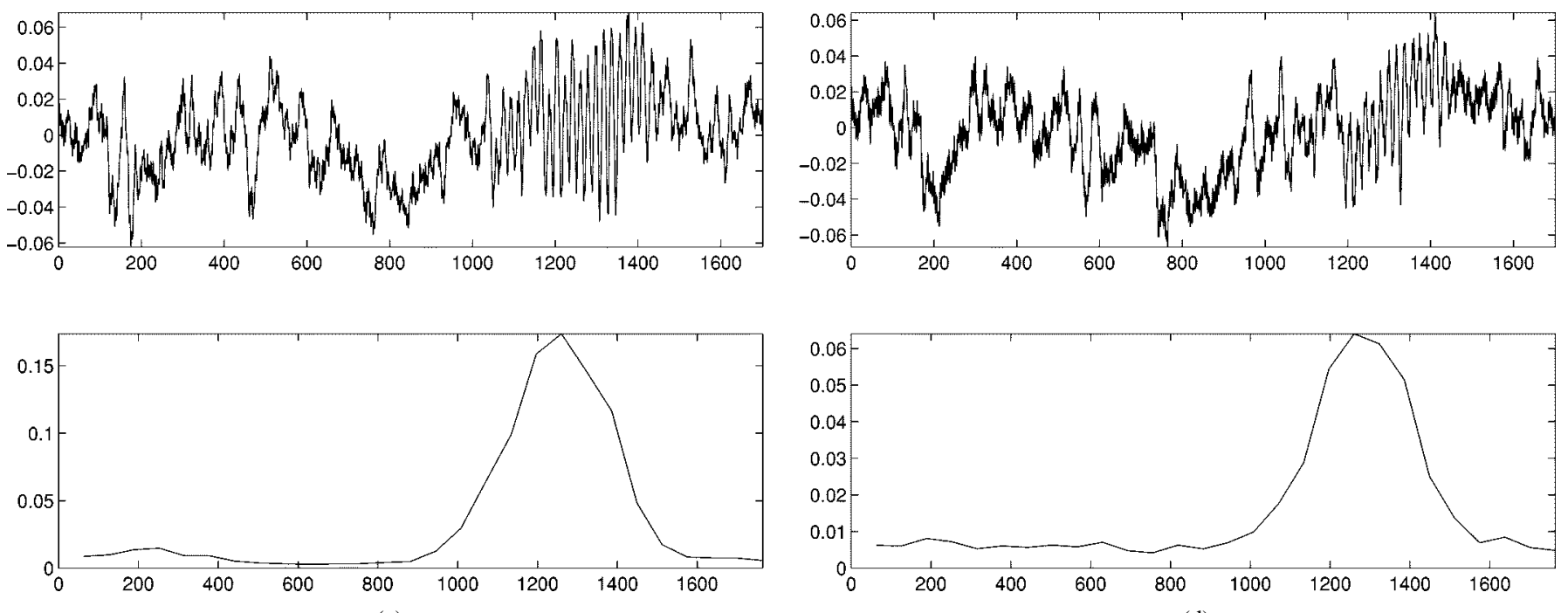

(c)

(d)

Fig. 2. Raw EEG samples of $\mathrm{Cz}, \mathrm{Fz}, \mathrm{Pz}$ and $\mathrm{Oz}$ (top plots). After applying a bandpass (8-15 Hz) filter to each channel their energy distributions over samples (bottom plots) in (a), (b), (c) and (d); respectively.

\section{B. Frequency Domain Results}

The averaged normalized bispectra and power spectra ${ }^{5}$ of sets $\mathrm{C}, \mathrm{F}$, and $\mathrm{P}$ from sample points 1135-1390 are given in Figs. 5(a) and (b)-7(a) and (b), respectively. In Fig. 5(a) the contour plot of the averaged normalized bispectrum from the channels of data in the set $\mathrm{C}$ is given, whereas in Fig. 5(b) of the same figure the averaged normalized power spectrum for the same set is presented. While the power spectrum exhibits a strong peak in the $12-15 \mathrm{~Hz}$ range, the normalized bispectrum reveals how tightly the frequency values interact quadractically among themselves. For example, as seen in Fig. 5(a), the frequency regions at $\left(f_{1}, f_{2}\right)$ where $f_{1}=12 \sim 14$ and $f_{2}=1 \sim 2$ $\mathrm{Hz} ; f_{1}=6 \sim 9$ and $f_{2}=4 \sim 6 \mathrm{~Hz} ; f_{1}=12 \sim 14$ and $f_{2}=13 \sim 14 \mathrm{~Hz}$; and finally $f_{1}=1.5$ and $f_{2}=4.5 \mathrm{~Hz}$ show strong (almost unity) quadratical interactions. However, it is quite difficult, if not impossible, to extract this information

${ }^{5}$ For visualization purposes, the averaged power spectrum values are normalized with respect to the peak value for each figure. Also, for the normalized bispectrum, only the half of the triangular region is shown. from Fig. 5(b) only. Fig. 5(a) clearly indicates more frequency interactions.

Similar quadratic interactions are observed for the set $F$ data in Fig. 6(a). Compared to the result of the previous set, the distribution of frequency values seems to be similar. However, the more numerous interactions are shifted toward $f_{1}+f_{2}=14$ $\mathrm{Hz}$ line and the region at $\left(f_{1}, f_{2}\right)$ where $f_{1}=12 \sim 14$ and $f_{2}=4 \sim 6 \mathrm{~Hz}$. The averaged and normalized power spectrum shown in Fig. 6(b) reveals additional low frequency activity when compared with the result of the previous set.

The results related to set $P$ is given in Fig. 7. It is clear that for this time period, this region of brain is highly dominated by the spindle activity as the bispectrum shown in Fig. 7(a) and the power spectrum shown in Fig. 7(b). Moreover, the bispectra suggest that it would be more realistic to think that the sleep spindle activity has at least some types of second-order nonlinearity (due to the appearance of the strong interactions in the $f_{1}=13-15$ and $f_{2}=12.5-14 \mathrm{~Hz}$ region). In addition, another interesting point arises when we compare the power spectrum estimate given in Fig. 5(b) with the one presented in Fig. 7(b). 
dahl5raw $-\mathrm{Cz}$
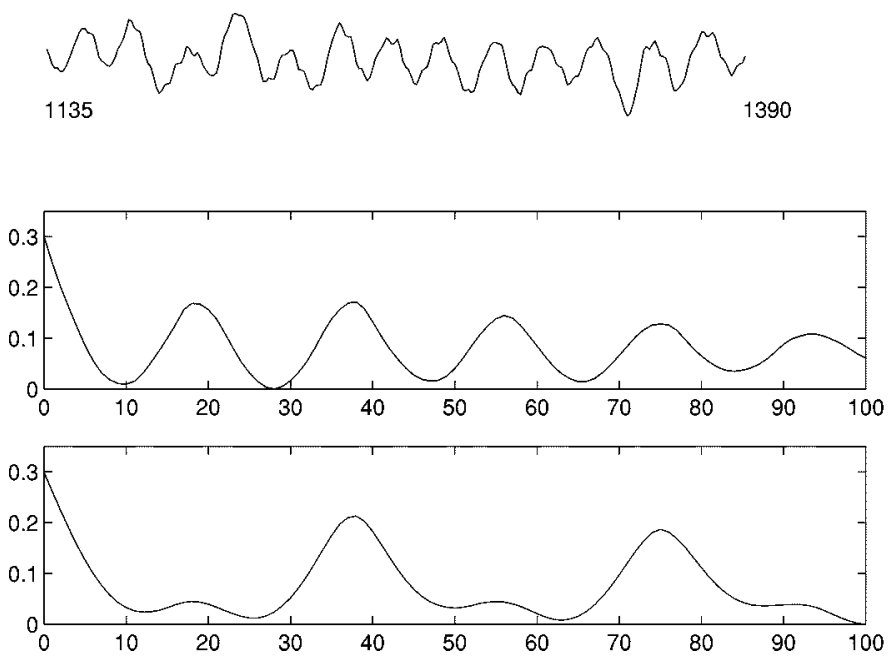

(a)

dahl5raw $-\mathrm{Pz}$

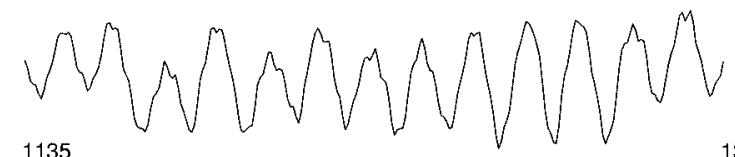

1135

1390
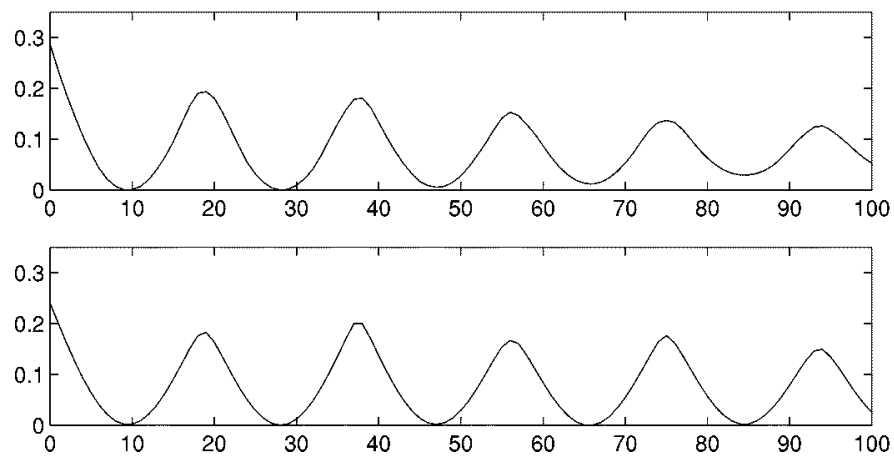

(c) dahl5raw $-\mathrm{Fz}$

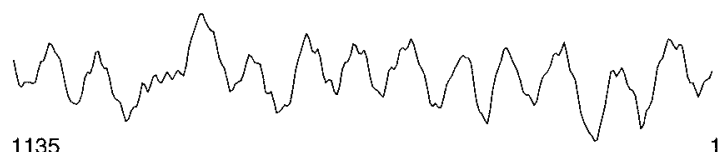

1135

1390
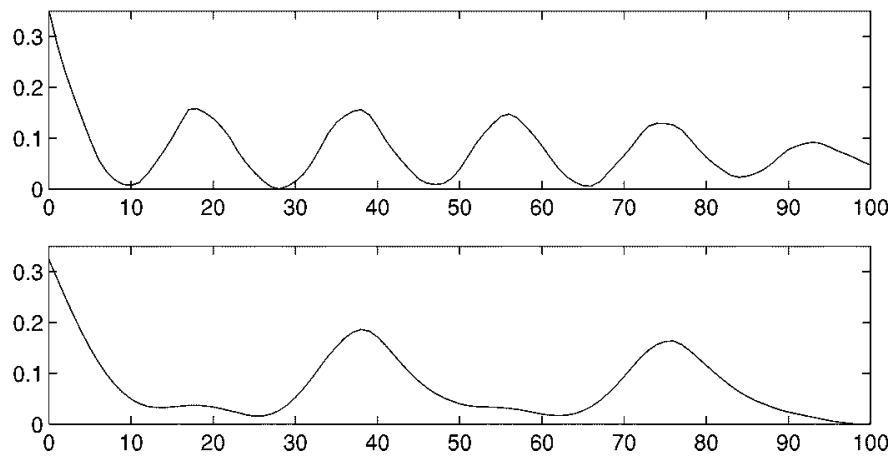

(b)

dahl5raw - Oz
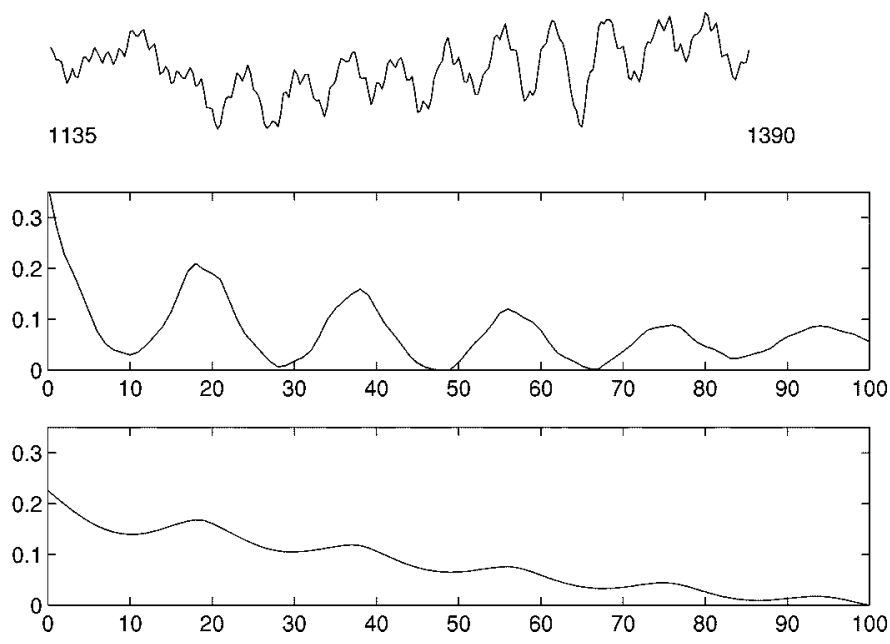

(d)

Fig. 3. EEG segments of $\mathrm{Cz}, \mathrm{Fz}, \mathrm{Pz}$ and $\mathrm{Oz}$ from samples 1135-1390 (top plots), mAMDF measurements (middle plots), mAMDFSoA of data with lag 100 (bottom plots) in (a), (b), (c), and (d); respectively.

Although they seemed to be similar, the bispectra in Fig. 5(a) and Fig. 7(a) are drastically different.

We show the results for sets C, F and P when the window is moved forward to cover samples 1198-1453 in Figs. 8(a) and (b)-10(a) and (b), respectively. In Fig. 8(a) the contour plot of the averaged normalized bispectrum from set $C$ is given, whereas in Fig. 8(b) normalized power spectrum is shown. The power spectrum continues to exhibit a strong peak in the 12-15 $\mathrm{Hz}$ range. The normalized bispectrum in Fig. 8(a), when compared to Fig. 5(a), indicates relatively weaker quadratical interactions in the $\left(f_{1}+f_{2}\right) \approx 13 \mathrm{~Hz}$ line. The existance of a lower frequency activity is visible both in the power spectrum and bispectrum plots.

Weaker quadratic interactions are observed for the set $\mathrm{F}$ data in Fig. 9(a) when compared to Fig. 6(a). Now, interactions are shifted toward the low frequency region. On the other hand, the averaged and normalized power spectra shown in Figs. 9(b) and 6(b) have similar results.
The results related with set $P$ are included in Fig. 10, where they confirm that the spindle activity is dominating this region of brain with showing small interactions with other lower frequency components.

\section{Test by Simulation}

To support the results of the time and frequency domain methods, we have simulated the spindle data by selecting a simple second-order nonlinear model, i.e.,

$$
y(n)=x(n)+K x^{2}(n)+g(n)
$$

where $K$ is a constant, $g(n)$ is noise and $x(n)$ is defined as

$$
x(n)=\sum_{i=1}^{L} a_{i} \sin \left(2 \pi f_{i} n\right) .
$$


dahl5raw $-\mathrm{Cz}$
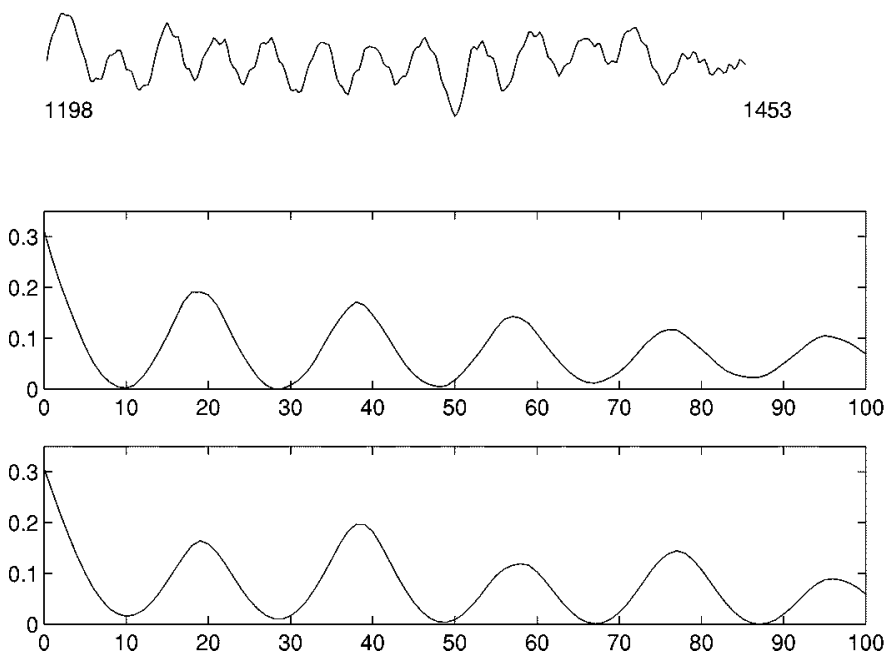

(a)

dah $\mid 5$ raw $-\mathrm{Pz}$

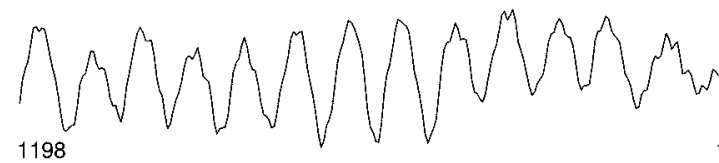

1453
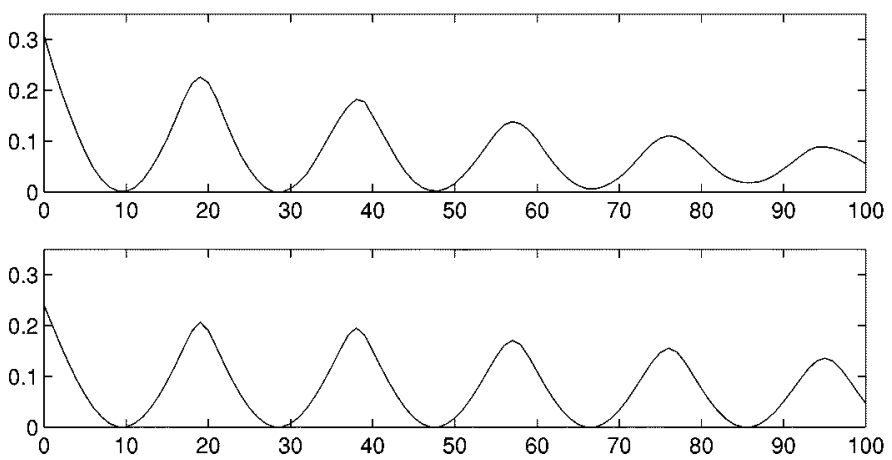

(c) dahl5raw $-\mathrm{Fz}$

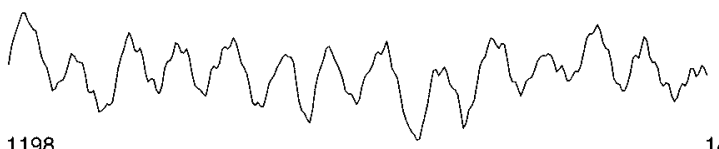

1198

1453
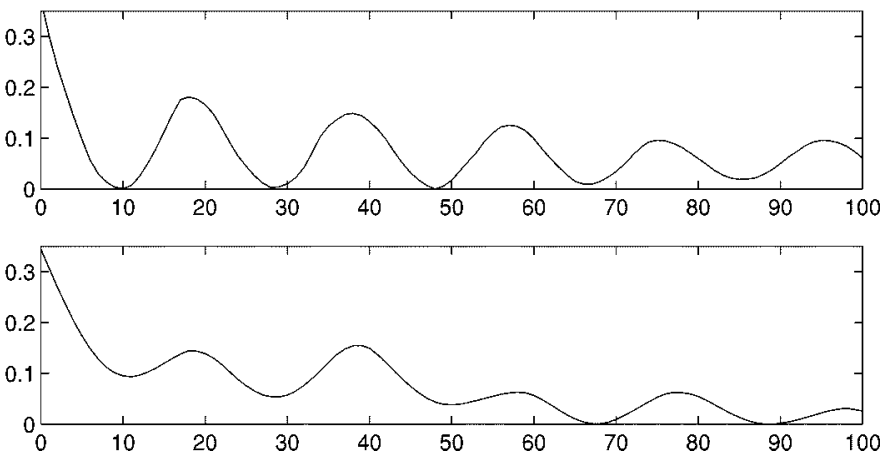

(b)

dahl5raw $-\mathrm{Oz}$

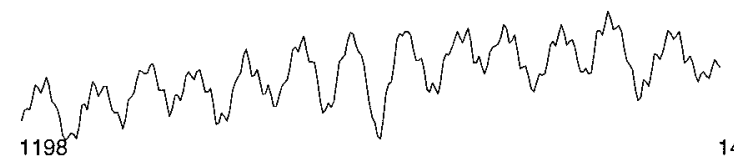

1453
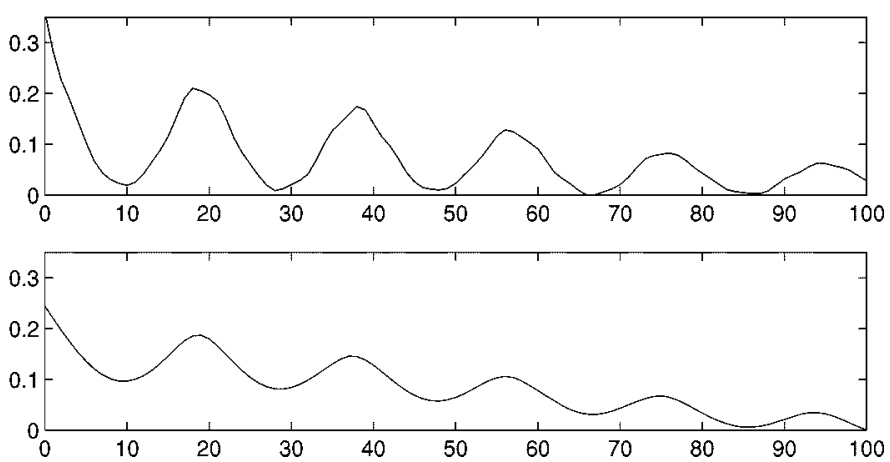

(d)

Fig. 4. EEG segments of $\mathrm{Cz}, \mathrm{Fz}, \mathrm{Pz}$ and $\mathrm{Oz}$ from samples 1198-1453 (top plots), mAMDF measurements (middle plots), mAMDFSoA of data with lag 100 (bottom plots) in (a), (b), (c), and (d); respectively.

In (17) $L$ is the number of dominant frequencies, $f_{i}$, in $\gamma, \theta, \alpha$ and $\beta$ bands, and $a_{i}$ 's are the corresponding amplitude values, respectively. For the purpose of demonstration, we assigned values to these parameters as

$$
\begin{aligned}
L & =5, \quad K=0.3 \\
\left\{f_{1}, f_{2}, f_{3}, f_{4}, f_{5}\right\} & =\{2,4,6,13,14\} \\
\left\{a_{1}, a_{2}, a_{3}, a_{4}, a_{5}\right\} & =\{0.3,0.24,0.22,0.28,1.0\}
\end{aligned}
$$

and added zero-mean Gaussian noise to maintain a signal-tonoise ratio of $20 \mathrm{~dB}$. We, then, estimated and plotted the normalized bispectrum, averaged power spectrum and mAMDF plus mAMDFSoA in Fig. 11(a)-(c). The normalized bispectrum in Fig. 11(a) reveals the nonlinearity through strong frequency in- teractions in $f_{2}=2, f_{1}+f_{2}=14$ and $f_{1}=14 \mathrm{~Hz}$ lines. In contrast, the nonlinearity cannot be detected by the power spectrum given in Fig. 11(b). It is interesting to observe that, for this data, the mAMDF and mAMDFSoA exhibit similar behavior as shown in Fig. 11(c).

\section{DISCUSSION}

Various Fourier transform-based frequency methods for data analysis have been applied to the sleep EEG for more than two decades [3], [23], [24]. However, the use of higher order spectral techniques (i.e., the normalized bispectrum) which may detect and measure the interactions between frequency components of the EEG has only been investigated in a few cases. 


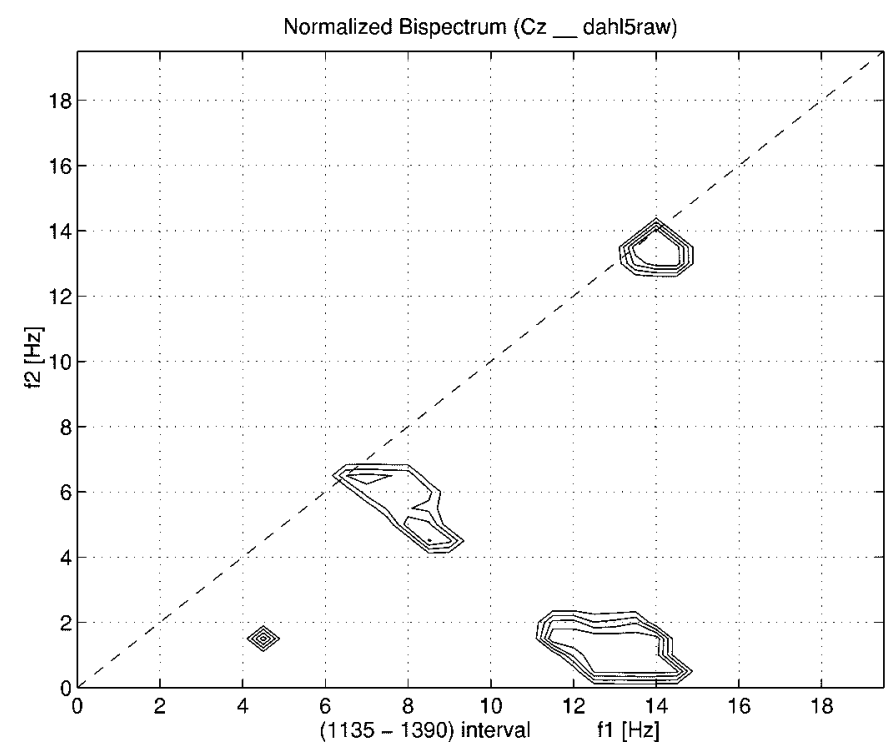

(a)

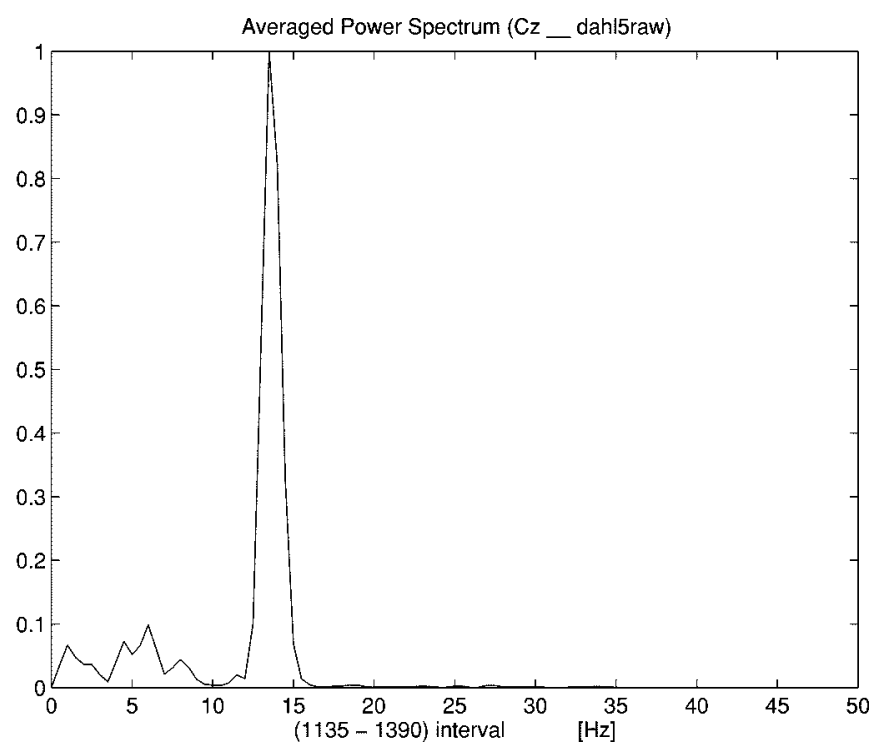

(b)

Fig. 5. For data set of Cz, C1, C2, C3, C4, C5, C6, C7 from samples 1135-1390. (a) Contour plot of the normalized bispectrum. (b) Averaged and normalized power spectrum.

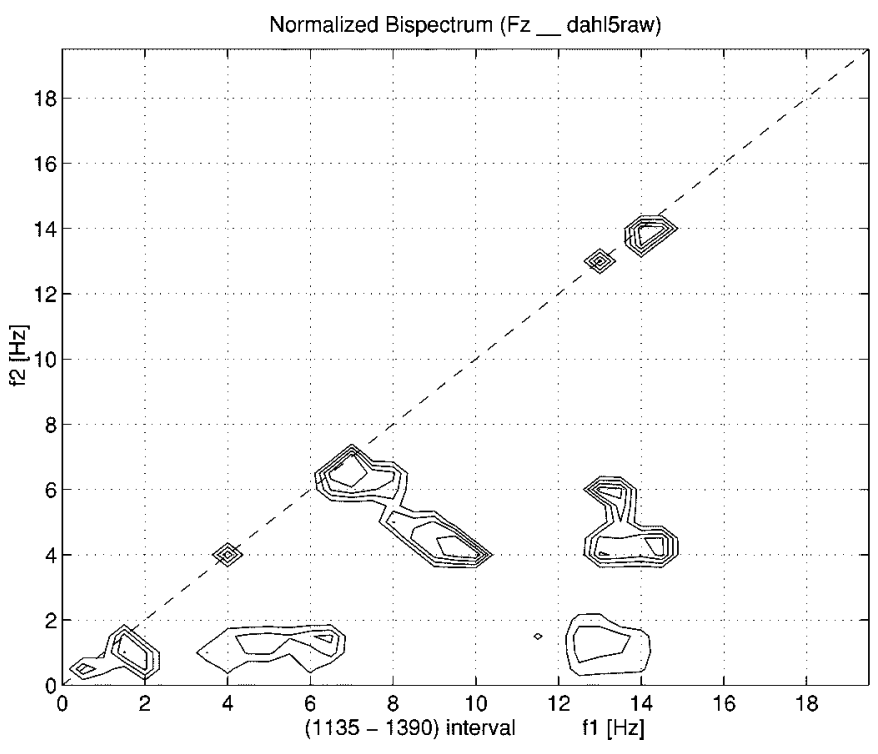

(a)

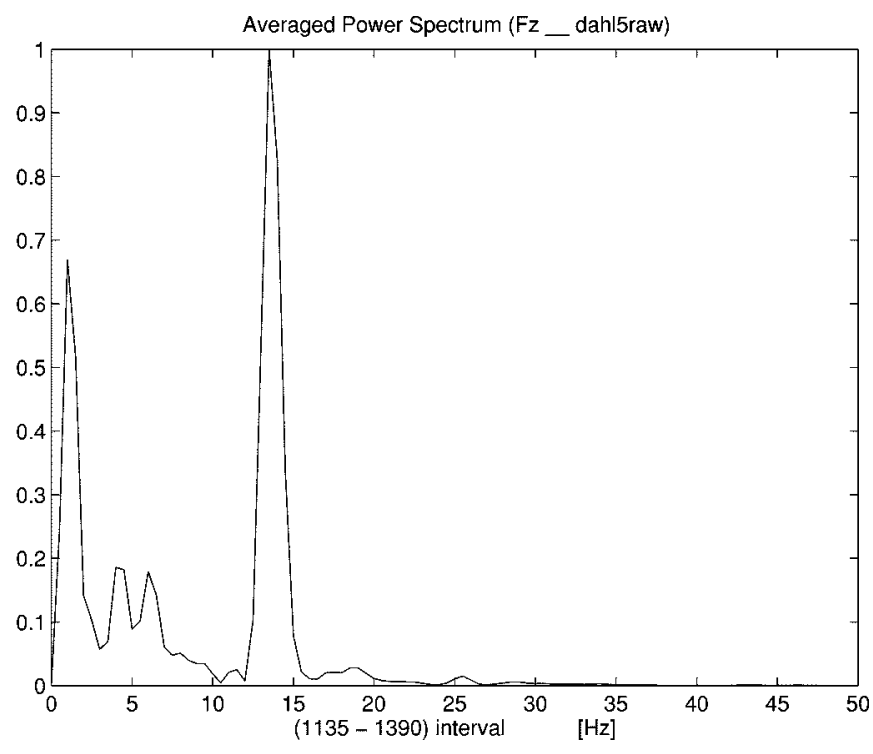

(b)

Fig. 6. For data set of Fz, F1, F2, F3, F4, F5, F6, F7, F8, F9 from samples 1135-1390. (a) Contour plot of the normalized bispectrum. (b) Averaged and normalized power spectrum.

One of the first applications of the bispectral analysis was the analysis of human EEG during sleep/wake states [25]. In this study, it was reported that, during the wake state, beta activity was dependent on alpha activity, that this dependency could be estimated by computing the bispectrum, and that this activity was not related to sleep stages. Another bispectral application of EEG was in the detection and quantification of the phase coupling in the cortical and hippocampal EEG in rats [26]. It was also demonstrated that the hippocampal EEG during REM sleep showed a strong quadratic phase coupling in $\theta$ rhythms [27]. Recently, a comparative study to the evaluation of the parametrical bispectral algorithms dealing with the identification of the alpha rhythms in EEG was reported [28]. Finally, a recent study investigated the measurement of linear and nonlinear phase relationships between EEG signals from different electrode positions in cortical areas during execution of finger movements [29].

In this study, we have investigated time and frequency domain methods for analyzing sleep spindles. The time domain methods depend on the combination of second- and third-order statistical tools to detect the oscillatory dynamics of the spindle activity. In particular, we used two types of estimates: the autocorrelation and average magnitude differentiated sum-of-autotriplecorrelations. If both these second- and third-order methods exhibit sim- 


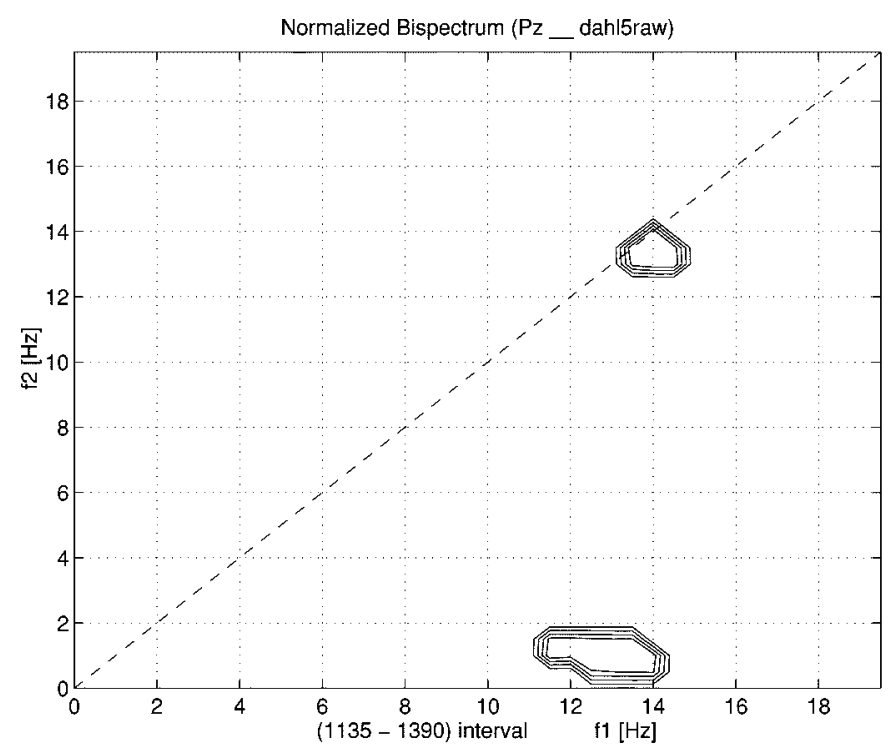

(a)

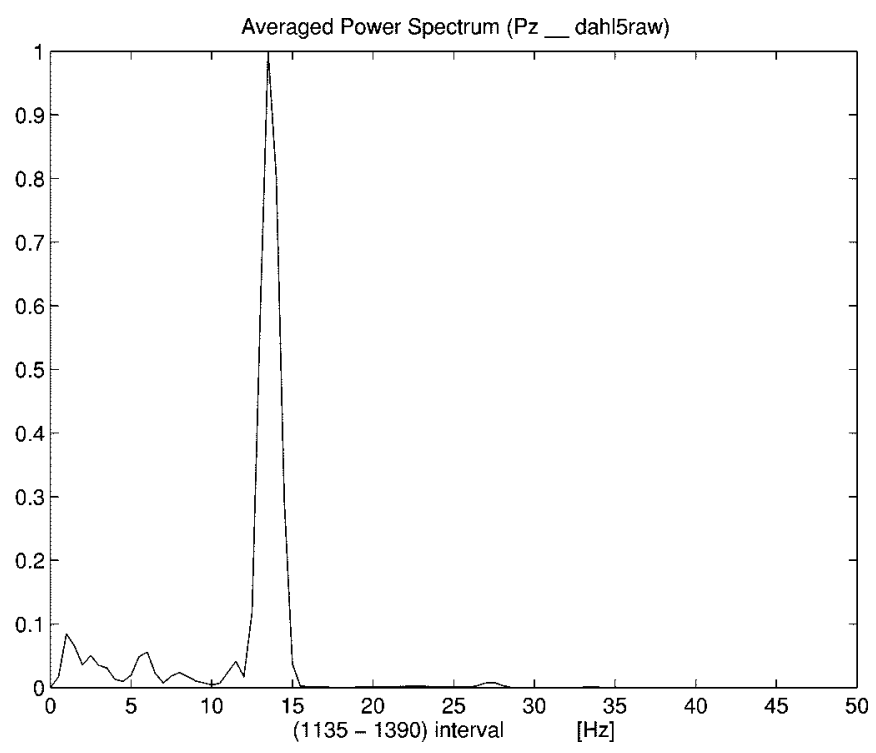

(b)

Fig. 7. For data set of Pz, P1, P2, P3, P4, P5, P6, P7, P8, P9 from samples 1135-1390. (a) Contour plot of the normalized bispectrum. (b) Averaged and normalized power spectrum.

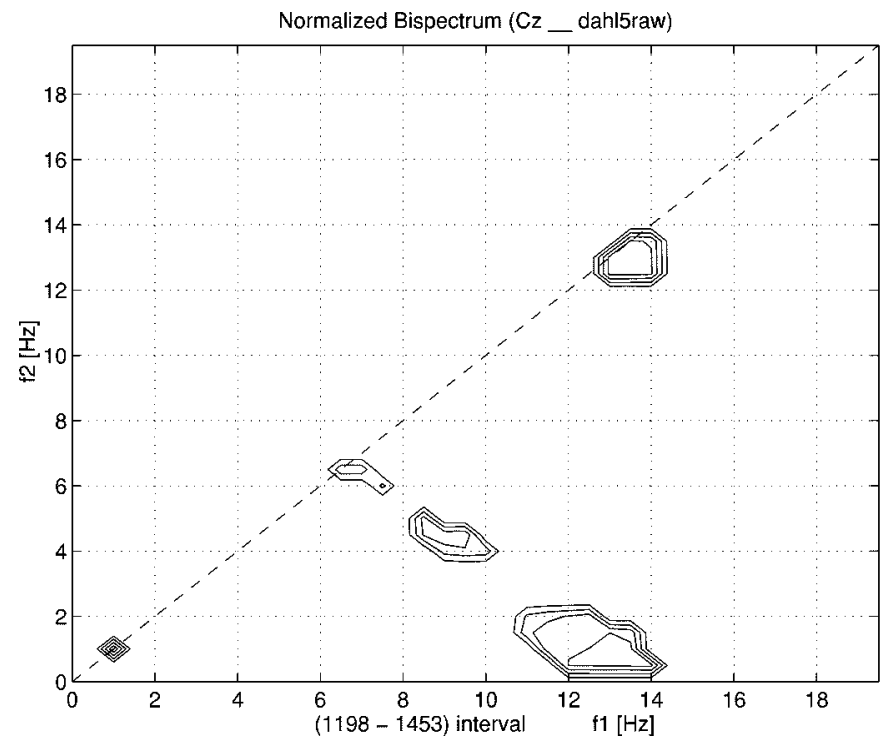

(a)

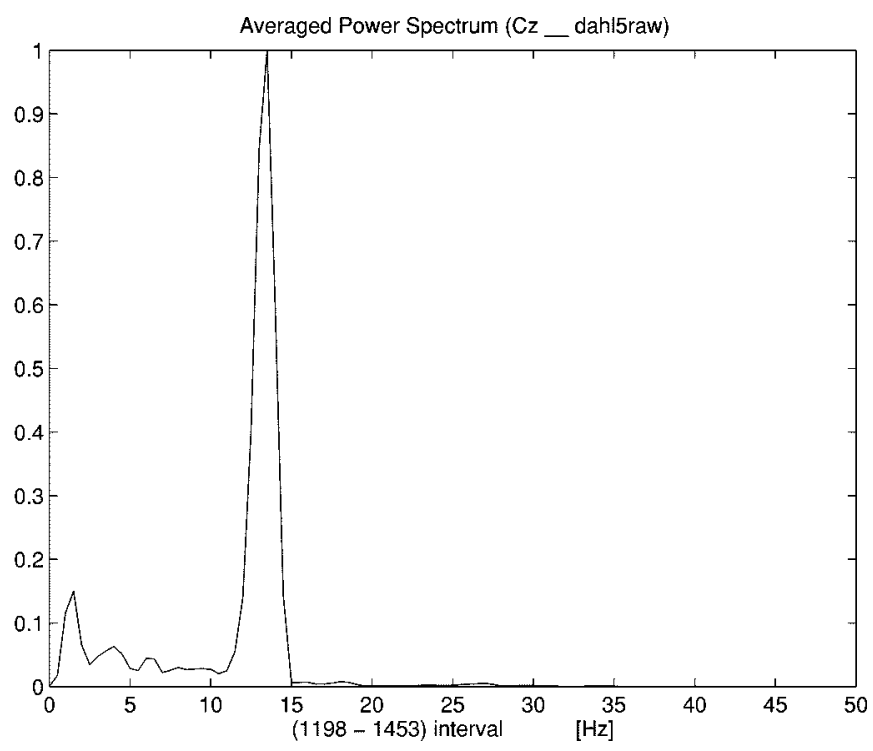

(b)

Fig. 8. For data set of Cz, C1, C2, C3, C4, C5, C6, C7 from samples 1198-1453. (a) Contour view of the normalized bispectrum. (b) Averaged and normalized power spectrum.

ilar periodic behavior then we conclude that only "stationary" spindle activity exists. However, if they are different then the data has some influence from other (linear or nonlinear) activities which may occur due to complexities within this particular frame [1], [30]. We want to emphasize here that these time-domain parameters are not useful for the detection of nonlinearities within given data, but for the detection of "stationarity" of the spindle oscillations.

On the other hand, the existence of a nonlinearity can be tested via the bispectral analysis of EEG which characterizes the interaction of activity (within selected EEG segments) for different frequencies. It is important to mention that the selec- tion of adequate EEG segments is critical, as it is necessary to assume that the characteristics of the signal are approximately constant over these segments. For the frequency domain method, we have summarized the estimation method of the normalized bispectrum and discussed the issues of detecting those quadratic couplings which may occur due to existing nonlinearities. We have applied the bispectral techniques to adequately grouped EEG sleep stages and different epochs of various EEG sleep recordings. Our results suggest that, during sleep spindle activity, some types of nonlinearities exist. However, since our tests were limited to identifying second-order nonlinearities, the existence of higher order $(>2)$ nonlinearities 


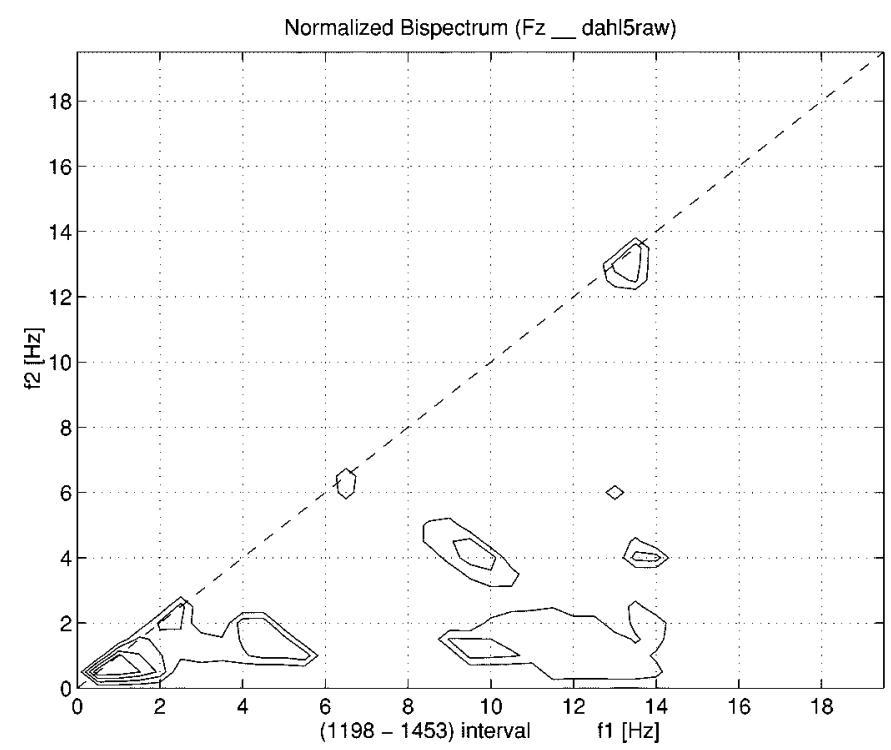

(a)

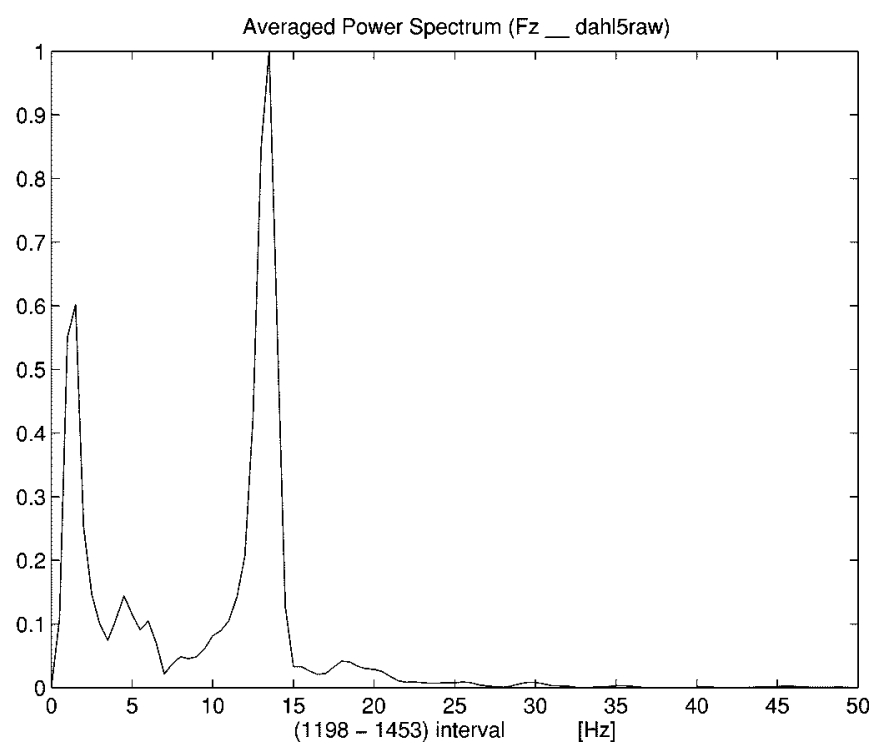

(b)

Fig. 9. For data set of Fz, F1, F2, F3, F4, F5, F6, F7, F8, F9 from samples 1198-1453. (a) Contour plot of the normalized bispectrum. (b) Averaged and normalized power spectrum.

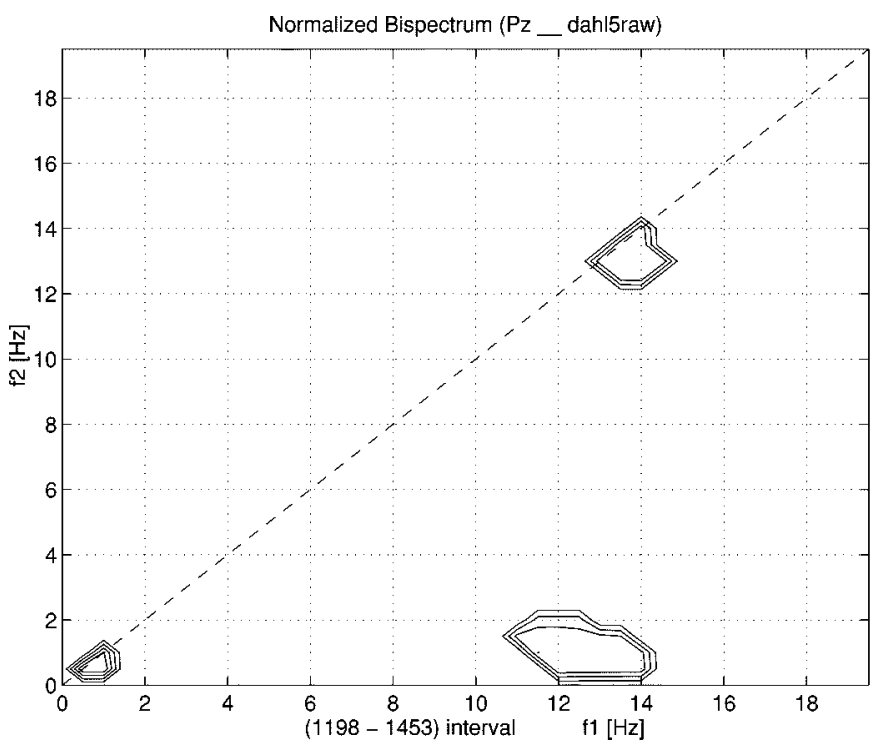

(a)

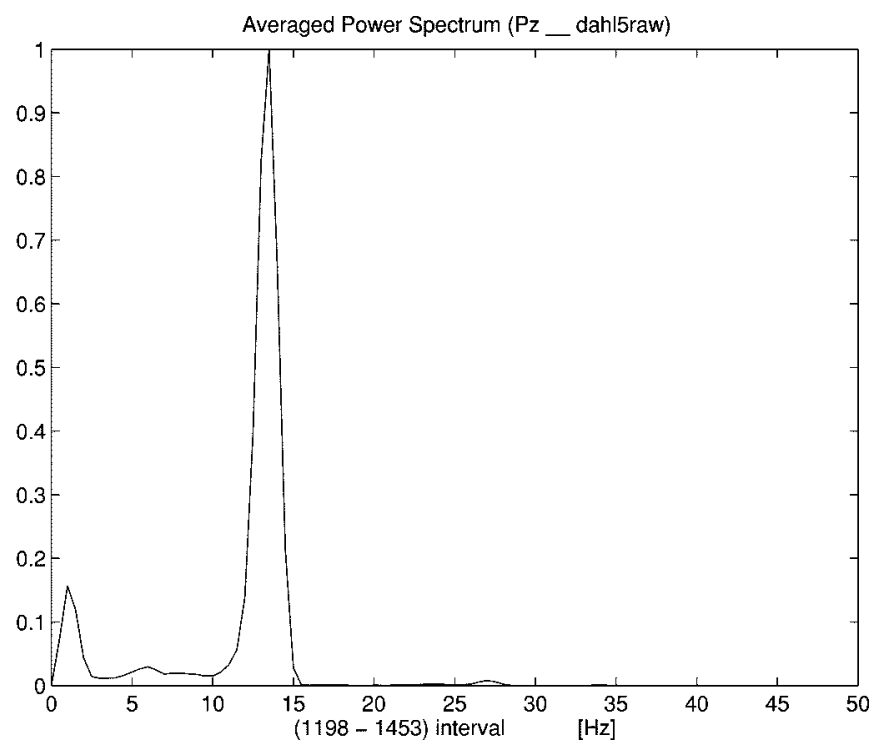

(b)

Fig. 10. For data set of Pz, P1, P2, P3, P4, P5, P6, P7, P8, P9 from samples 1198-1453. (a) Contour plot of the normalized bispectrum. (b) Averaged and normalized power spectrum.

should be checked for all possible orders of the normalized higher order spectra.

In many applications, bispectral estimates in (4) can be adequately used for detection of periodicities [31], (9) acts as the discriminant of linear from nonlinear processes. For example, $b^{2}$ is constant either for linear systems ${ }^{6}$ [15] or fully coupled frequencies [32] and $b^{2}=0$ for either Gaussian signals ${ }^{7}$ or random phase relations where no quadratic couplings occur. Clearly,

${ }^{6}$ However, constant $b^{2}\left(\omega_{1}, \omega_{2}\right)$ does not guarantee a linear system.

${ }^{7}$ In theory, since the bispectrum of zero-mean Gaussian process is zero, the normalized bispectrum is also null which is a measure for the EEG segments whether they are Gaussian (symmetrically distributed) or not. when the values of normalized bispectrum vary between zero and one $\left(0<b^{2}<1\right)$, then couplings of the frequencies occur. The coherency value that is close to unity at $\left(\omega_{i}, \omega_{j}\right)$ frequency pairs indicates a quadratic interaction; however, a value close to zero indicates either low or absent interactions [14]. Nevertheless, in general, there may be couplings occuring at various frequency values where the transfer of energy is not due to $\left(\omega_{i}+\omega_{j}\right)$ only, so this interpretation is rather difficult or even invalid [32]. Although such a case is complicated and difficult to interpret, the normalized bispectrum suggests the degree of couplings between two frequencies. For the statistics of the normalized bispectral estimate given in (9), the interested reader 


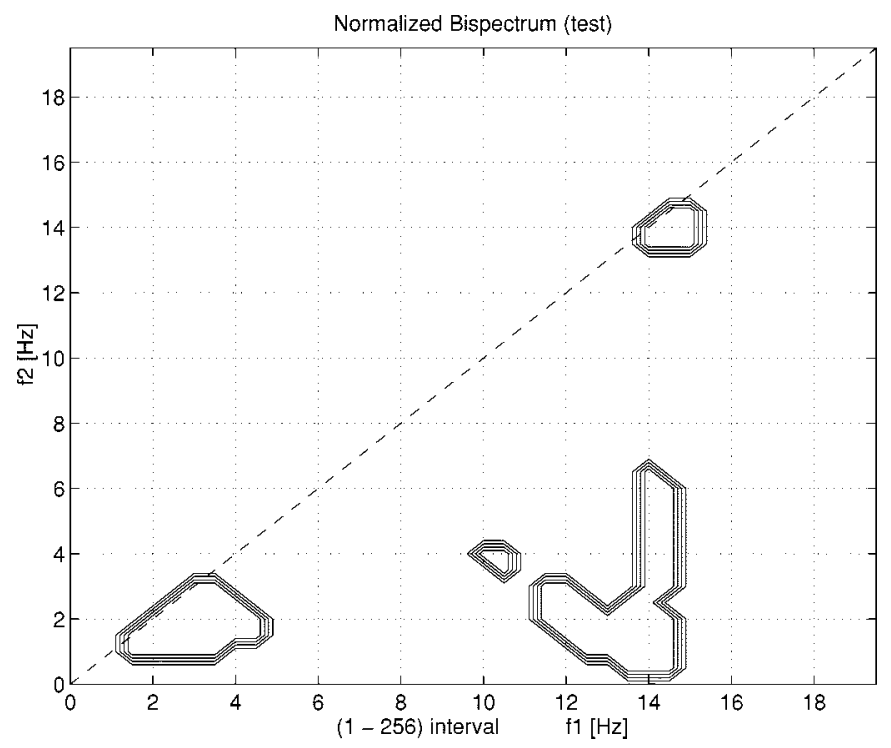

(a)

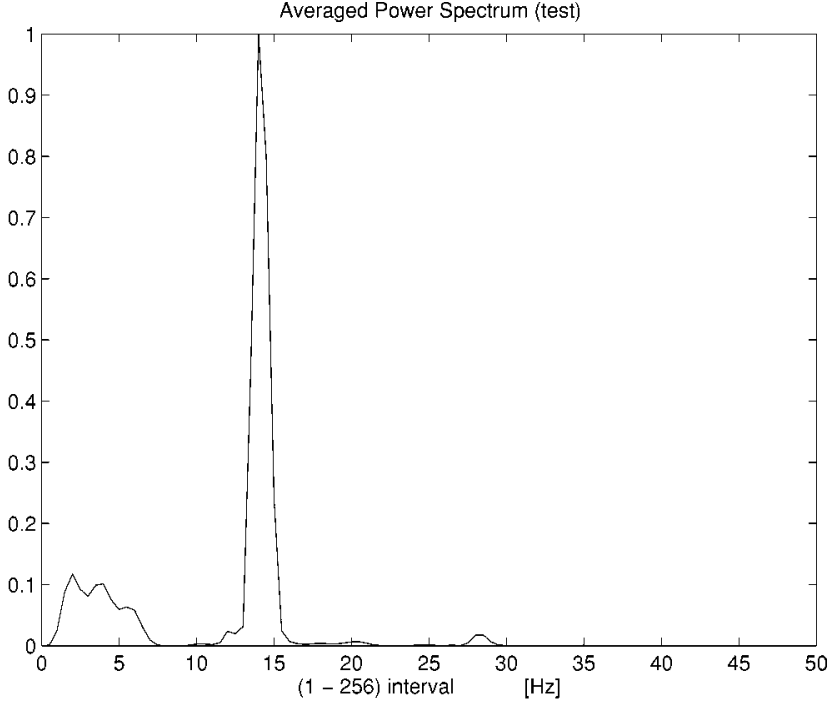

(b)

test
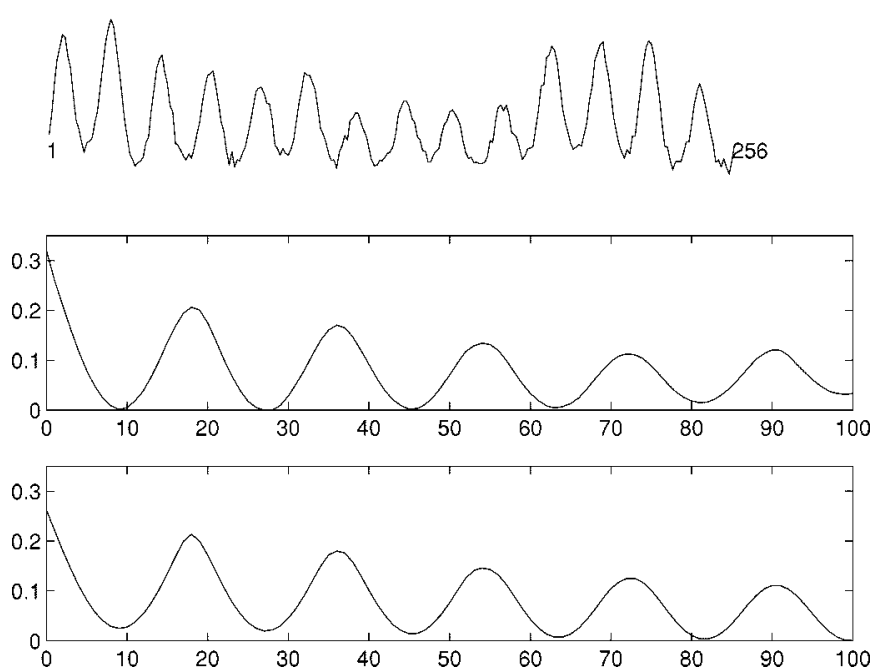

(c)

Fig. 11. For artificial data. (a) Contour plot of the normalized bispectrum; (b) averaged and normalized power spectrum. (c) its mAMDF (middle) and its mAMDFSoA (bottom).

may study [33] and the references therein, as well as [34] to overcome the confusion which may arise during practical work. The bispectrum also suppresses the zero-mean additive noise if the noise is uncorrelated to the signal and symmetrically distributed [13], [14]. However, in practice, one should pay attention to noise contamination, which may corrupt the linearity and nonlinearity test results [35].

Both the time and frequency domain experiments show that: 1) spindle activity may not uniformly dominate all regions of brain; 2) during the spindle activity frontal recordings still exhibit rich mixtures in frequency contents and couplings. On the other hand, data from the posterior region of the head exhibit a poor couplings but demonstrate dominancy to spindle activity, which confirms some other recent (second-order statistics based) studies [6]; 3) evidences suggest that sleep spindle activity ought to be envisaged by having at least second-order nonlinearity.

\section{ACKNOWLEDGMENT}

The authors would like to thank the anonymous reviewers for their constructive comments.

\section{REFERENCES}

[1] E. Başar, Chaos in Brain Function, E. Başar, Ed. Berlin, Germany: Springer-Verlag, 1990.

[2] A. A. Borbely and P. Achermann, "Concepts and models of sleep regulation: An overview,” J. Sleep Res., vol. 1, pp. 63-79, 1992.

[3] R. M. Harper, R. J. Sclabassi, and T. Estrin, "Time series analysis and sleep research," IEEE Trans. Automat. Contr., vol. AC-19, no. 6, pp. 932-943, 1974.

[4] E. Niedermeyer and F. E. Lopes da Silva, Electroencephalography, E. Niedermeyer and F. E. Lopes da Silva, Eds. Baltimore, MD: Williams and Wilkins, 1993.

[5] M. Steriade, E. G. Jones, and R. R. Llinas, Thalamic Oscillations and Signaling. New York: Wiley, 1990.

[6] J. Marc, E. Poiseau, P. Jähnig, H. Schulz, and S. Kubicki, "Topographical analysis of sleep spindle activity," Neuropsychobiology, vol. 26, pp. 210-217, 1992. 
[7] S. Uchida, T. Maloney, J. D. March, R. Azari, and I. Feinberg, "Sigma $(12-15 \mathrm{~Hz})$ and delta $(0.3-3)$ EEG oscillate reciprocally within NREM sleep," Brain Res. Bull., no. 27, pp. 93-96, 1991.

[8] M. Lancel, H. van Riezen, and A. Glatt, "The time course of sigma activity and slow-wave activity during NREMS in cortical and thalamic EEG of the cat during baseline and after 12 hours of wakefulness," Brain Res., no. 596, pp. 285-295, 1992.

[9] M. Sun, S. Qian, X. Yan, S. B. Baumann, X. Xia, R. E. Dahl, N. D. Ryan, and R. J. Sclabassi, "Localizing functional activity in the brain through time-frequency analysis and synthesis of the EEG," Proc. IEEE, vol. 64, pp. 1302-1311, Sept. 1996.

[10] P. L. Nunez, Neocortical Dynamics and Human EEG Rhythms. New York: Oxford Univ. Press, 1995.

[11] D. R. Brillinger, "An introduction to polyspectra," Ann. Math. Stat., vol. 36, pp. 1351-1374, 1965

[12] L. C. Nikias and M. R. Raghuveer, "Bispectrum estimation: A digital signal processing framework," Proc. IEEE, vol. 75, pp. 869-891, July 1987.

[13] J. M. Mendel, "Tutorial on higher order statistics (spectra) in signal processing and system theory: Theoretical results and some applications," Proc. IEEE, vol. 79, pp. 278-305, Mar. 1991.

[14] C. L. Nikias and A. Petropulu, Higher Order Spectra Analysis: A Nonlinear Signal Processing Framework. Englewood Cliffs, NJ: PrenticeHall, 1993.

[15] T. S. Rao, "Bispectral analysis of nonlinear stationary time series," in Handbook of Statistics 3, D. R. Brillinger and P. R. Krishnaiah, Eds. Amsterdam, The Netherlands: North Holland, 1983.

[16] L. R. Rabiner and R. W. Schafer, Digital Processing of Speech Signals. Englewood Cliffs, NJ: Prentice-Hall, 1978.

[17] S. Alshebeili, A. E. Cetin, and A. N. Venetsanopoulos, "Identification of nonminimum phase MA systems using cepstral operations on slices of higher order spectra," IEEE Trans. Circuits Syst., vol. 39, no. 9, pp. 634-637, 1992.

[18] T. Akgül, A. El-Jaroudi, and M. Simaan, "Multi-scale deconvolution of sensor array signals via sum-of-cumulants," IEEE Trans. Signal Processing, vol. 45, pp. 1656-1659, June 1997.

[19] T. Akgül and A. El-Jaroudi, "Reconstruction of mixed-phase signals from sum-of-auto-triplecorrelations," IEEE Trans. Signal Processing, vol. 46, pp. 250-254, Jan. 1998.

[20] E. R. Kandel, J. H. Schwartz, and T. M. Jessell, Principles of Neural Science, 3rd ed. New York: Elsevier/North Holland, 1991.

[21] W. W. Orrison, Jr., J. D. Lewine, J. A. Sanders, and M. F. Hartshorne, Functional Brain Imaging. St. Louis, MO: Mosby-Year Book, 1995, ch. 8, pp. 327-368.

[22] R. J. Sclabassi, M. Sun, D. N. Krieger, P. Jasiukaitis, and M. S. Scher, "Time-frequency domain problems in the neurosciences," in Time-Frequency Signal Analysis: Methods and Applications, B. Boashash, Ed, U.K.: Wiley Halsted, 1992, pp. 498-519.

[23] B. W. Jervis, M. Coelho, and G. W. Morgan, "Spectral analysis of EEG responses," Med. Biol. Eng. Comput., vol. 27, pp. 230-238, 1989.

[24] R. J. Sclabassi and R. M. Harper, "Laboratory computers in neurophysiology," Proc. IEEE, vol. 61, pp. 1602-1614, Nov. 1973

[25] T. P. Barnett, L. C. Johnson, P. Naitoh, N. Hicks, and C. Nute, "Bispectrum analysis of electroencephalogram signals during waking and sleeping," Science, vol. 172, no. 3981, pp. 401-402, 1971.

[26] T. Ning and J. D. Bronzino, "Bispectral analysis of the rat EEG during various vigilance stages," IEEE Trans. Biomed. Eng., vol. 36, pp. 497-499, Apr. 1989.

[27] _ "Nonlinear analysis of the hippocampal subfields of CA1 and the Dentate gyrus," IEEE Trans. Biomed. Eng., vol. 40, pp. 870-876, Sept. 1993.

[28] D. L. Sherman and M. D. Zoltowski, "Decomposing the alpha rhythms: Comparative performance evaluation of parametric bispectral algorithms for EEG," in Proc. 6th SSAP Workshop, 1992, pp. 522-525.

[29] G. Edlinger, C. Andrew, and G. Pfurtscheller, "Second order and third order statistics with application to EEG," in Proc. IEEE Medicine and Biology Conf., vol. 2, 1995, pp. 879-890.

[30] J. Röschke and J. B. Aldenhoff, "A nonlinear approach to brain function: Deterministic chaos and sleep EEG," Sleep, vol. 15, no. 2, pp. 95-101, 1992.

[31] T. S. Rao and M. M. Gabr, "The estimate of the bispectral density function and the detection of periodicities in a signal," J. Multivariate Anal., no. 27 , pp. $457-477,1988$

[32] O. Michel and P. Flandrin, "Higher order statistics for chaotic signal analysis," in Volumes on DSP Techniques and Applications, E. T. Leondes, Ed. New York: Academic, 1995.
[33] G. Sebert and S. Elgar, "Statistics of bicoherence and biphase," in Proc. IEEE Workshop on Higher Order Spectral Analysis, 1989, pp. 223-228.

[34] A. Swami, "The pitfalls in polyspectra," in Proc. ICASSP-93, 1993, pp. $97-100$

[35] J. K. Tugnait, "Testing for linearity of noisy stationary signals," IEEE Trans. Signal Processing, vol. 42, pp. 2742-2748, Oct. 1994.

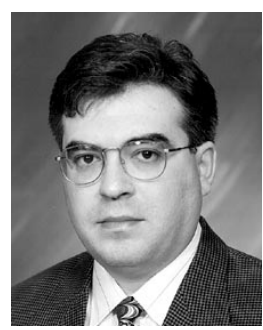

Tayfun Akgül (M'94) received the B.S. and M.S. degrees in electrical engineering in 1985 and 1988 from Istanbul Technical University, Istanbul, Turkey, and the $\mathrm{Ph} . \mathrm{D}$. degree in electrical engineering in 1994 from the University of Pittsburgh, Pittsburgh, PA.

Between March 1986 and August 1988, he was a Research Engineer in the Scientific and Technical Research Council of Turkey (TUBITAK). In September 1988, he joined the Department of Electrical and Electronics Engineering, Çukurova University, Adana, Turkey, as an Assistant. From September 1989 to April 1994, he was with the Real-Time Signal Processing and Computational Neuroscience laboratories at the University of Pittsburgh. From June 1994 to July 1996, he was an Assistant Professor and then Associate Professor in the Department of Electrical and Electronics Engineering, Cukurova University. He was TUBITAK-BAYG postdoctoral fellow in the Laboratory for Computational Neuroscience, Departments of Neurological Surgery and Electrical Engineering, University of Pittsburgh in 1996. From April 1997 to September 1999, he was a Visiting Assistant Professor in the Electrical and Computer Engineering Department and the School of Biomedical Engineering, Science and Health Systems at Drexel University, Pittsburh, PA. Currently, he is a Research Associate Professor at Drexel University and Senior Researcher in the Information Technologies Research Institute at TUBITAK Marmara Research Center, Turkey.

Dr. Akgul is a member of Sigma Xi and the Chamber of Electrical Engineers of Turkey. He is also an active member of the Professional Cartoonists' Association of Turkey.

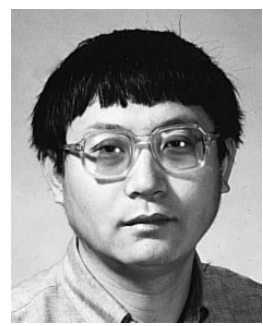

Mingui Sun (S'88-M'89) received the B.S. degree from the Shenyang chemical engineering Institute, Shenyang, China, in 1982, and M.S. and Ph.D. degrees in electrical engineering from the University of Pittsburgh, Pittsburgh, PA, in 1986 and 1989, respectively.

He held various positions in mechanical, electrical, and electronics engineering from 1971 to 1978 with the Chifeng Radio Elements, Inc., China prior to his undergraduate education. He was a Graduate Student Researcher from 1985 to 1989 working on signal and image processing projects. Currently, he is a Associate Professor and an Associate Director of the Center for Clinical Neurophysiology in the Department of Neurosurgery at the University of Pittsburgh. His current research interests include advanced biomedical devices, biomedical signal and image processing, artificial neural networks, wavelet transforms, time-frequency analysis, and the inverse problem of neurophysiological signals. He has over 100 publications in these areas.

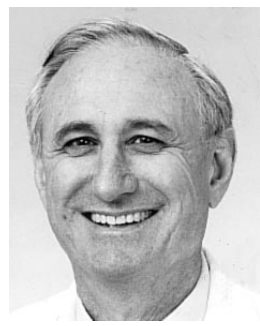

Robert J. Sclabassi (M'62-S'68-M'73-SM'92) received the B.S.E. degree from Loyola University, Los Angeles, CA, the M.S.E.E., Engr's, and Ph.D. degrees in electrical engineering from the University of Southern California, Los Angeles, and the M.D. degree from the University of Pittsburgh, Pittsburgh, PA.

He was employed in the Advanced Systems Laboratory at TRW, Los Angeles, CA, and was a postdoctoral fellow at The Brain Research Institute at The University of California, Los Angeles. He is currently a Professor of Neurological Surgery, Psychiatry, Neuroscience, Electrical Engineering, Mechanical Engineering, and Biomedical Engineering at The University of Pittsburgh.

Dr. Sclabassi is a Registered Professional Engineer 


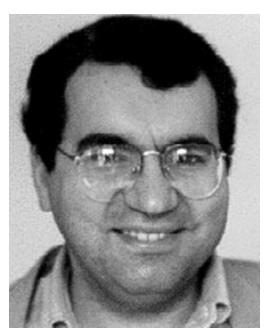

A. Enis Çetin (S'85-M'87-SM'95) studied electrical engineering at the Middle East Technical University, Ankara, Turkey. After receiving the B.Sc. degree, he received the M.S.E and Ph.D. degrees in systems engineering from the Moore School of Electrical Engineering, University of Pennsylvania, Philadelphia.

Between 1987-1989, he was Assistant Professor of Electrical Engineering at the University of Toronto, Toronto, ON, Canada. Since then he has been with Bilkent University, Ankara, Turkey. Currently he is a Full Professor. During summers of 1988, 1991, 1992 he was with Bell Communications Research (Bellcore), Morristown, NJ. He spent 1994-1995 academic year at Koc University, Istanbul, Turkey, and 1996-1997 academic year at the University of Minnesota, Minneapolis as a Visiting Associate Professor. Currently, he is the Director of the Picture Archiving and Communication Systems (PACS) Laboratory and a member of the Center for Turkish Language and Speech Processing (CTLSP).

Prof. Çetin received the young scientist award of TUBITAK (Turkish Scientific and Technical Research Council) in 1993. He serves as an Associate Editor of the IEEE TRANSACTIONS ON IMAGE PROCESSING, and a member of the DSP technical committee of the IEEE Circuits and Systems Society. He founded the Turkish Chapter of the IEEE Signal Processing Society in 1991. He is a senior member of EURASIP. He was the chair of the IEEE-EURASIP Nonlinear Signal and Image Processing Workshop (NSIP'99) which was held in June 1999 in Antalya, Turkey. 